\title{
RESIDÊNCIA MÉDICA EM NEUROLOGIA INFANTIL NO BRASIL
}

\author{
MARIA VALERIANA L. DE MOURA-RIBEIRO*, \\ CARMEN SILVIA SANCHES ${ }^{* *}$ SYLVIA MARIA CIASCA***
}

\begin{abstract}
RESUMO - Apresentamos os resultados de pequisa sobre Residência Médica em Neurologia Infantil, catalogando dados atualizados sobre o ensino e treinamento nos vários centros de formação do Brasil. Foi possível constatar 17 Instituições com programação organizadas, sendo 6 credenciadas pela CNRM, 10 não credenciadas e, um em diligência. O conteúdo programático é desenvolvido em 3 ou 4 anos, incluindo o pré-requisíto, sendo a carga horária anual, variável, de 1900 a 2880 horas/ano.
\end{abstract}

PALAVRAS-CHAVE: neurologia infantil, residência médica, Brasil.

\section{Medical residence in pediatric neurology in Brazil}

ABSTRACT - We present the results of a research on Medical Residence in Pediatric Neurology, classifying present information on the teaching and training in the several centers of formation in Brazil. It was possible to contact 17 Institutions with organized services, being 6 accredited by CNRM (National Council of Medical residence), 10 non accredited, and one under diligence. The program content is developed in 3 or 4 years, including the pre-qualification, being the annual schedule load variable, from1900 to 2880 hours / year.

KEY WORDS: teaching, pediatric neurology, medical residence, Brazil.

Na organização da estrutura geral de centros de ensino e treinamento em Residência Médica, se impõe um conjunto de exigências e requisitos mínimos que visam à aquisição de competências para o exercício profissional adequado ${ }^{1}$. No referente à Neurologia e Neurologia Infantil tem sido possível reunir professores dirigentes de centros de ensino, para discussão e elaboração de Documentos de Trabalho incluindo o conteúdo programático, distribuído em três ou mais anos. Nessa tarefa, a Comissão de Ensino da Academia Brasileira de Neurologia tem apresentado contribuição de relevancia, discutindo organização de recursos para qualificação das necessidades institucionais $^{1-3}$.

A CNRM (Comissão Nacional de Residência Médica) com competência para credenciar, organizar e avaliar programas no Brasil é o orgão responsável pelo controle da formação complementar do médico visando às necessárias garantias na sua capacitação profissional ${ }^{4}$. Assim, o conteúdo programático, a qualificação dos docentes, infra-estrutura, carga horária mínima, instalações com incorporação dos avanços tecnológicos e remuneração, devem ser regularmente fiscalizados ${ }^{1,2}$. Através de ciclo de seminários sobre competências mínimas em especialidades médicas, a FUNDAP (Fundação do Desenvolvimento Administrativo) tem desenvolvido, oficialmente, importante trabalho junto aos supervisores de programas das várias escolas médicas ${ }^{1}$. Dessa forma, os centros de

Disciplina de Neurologia Infantil do Departamento de Neurologia da Faculdade de Ciências Médicas da Universidade Estadual de Campinas (FCM) (UNICAMP):*Professor Associado;**Professor Assistente;***Professor Assistente Doutor. Aceite: 5-julho-2000.

Dra. Maria Valeriana L. de Moura Ribeiro - Departamento de Neurologia FCM, UNICAMP - Caixa Postal 6111 - 13083-970 Campinas, SP - Brasil.FAX 19788 7810. E-mail: valerianaribeiro@uol.com.br 
treinamento e formação se mantêm adequados a essas orientações, melhorando continuadamente as condições de trabalho visando ao credenciamento desejável e, o recredenciamento, periodicamente avaliado. Levando em conta críticas pertinentes,os benefícios desse modelo têm sido aceitos e valorizados, de tal forma que os egressos dos cursos médicos almejam a aprovação em concursos públicos realizados pelos centros de formação credenciados pela $\mathrm{CNRM}^{5-7}$.

A proposta deste estudo é apresentar uma visão atual do ensino e treinamento em Neurologia Infantil no Brasil.

\section{MÉTODO}

As informações registradas foram obtidas em uma primeira etapa (1992) através de cartas dirigidas aos Centros Universitários, Faculdades de Medicina e, outras Instituições dos vários estados da União envolvendo, também, os docentes responsáveis dos vários serviços de treinamento em Residência Médica.

Obtidas as informações relativas ao ensino em Residência Médica, em Neurologia, em Pediatria e, em Neurologia Infantil, encaminhamos às Instituições Universitárias e Faculdades de Medicina, em 1993 e 1994, um protocolo padrão para preenchimento, contendo questões básicas, relacionadas ao treinamento em serviço, visando obter dados objetivos sobre Residência Médica em Neurologia Infantil.

Em uma segunda etapa, nos anos de 1998-1999, procedemos a revisão e atualização dos dados, consultando diretamente as Instituições que mantiveram o ensino e treinamento de médicos interessados em Neurologia Infantil em conjunto com as informações obtidas na CNRM.

Assim, foram catalogados os dados sobre credenciamento, ou não, dos programas junto à CNRM, ano de credenciamento, pré-requisitos, número de vagas, época de seleção, duração de programas, carga horária anual, conteúdo programático. Também foram analisados os elementos relativos ao Corpo Docente (número, titulação, linhas de pesquisas), infra-estrutura (biblioteca, informatização, serviço de secretaría) e, como complementação, informações referentes ao treinamento em serviço de profissionais da àrea de saúde não médicos (psicólogos, psicopedagogos, fonoaudiólogos, terapeuta ocupacional, fisioterapeuta, entre outros). Portanto, os dados aqui registrados, foram conferidos diretamente junto aos docentes supervisores dos Programas de Residência em Neurologia Infantil das várias Instituições do Brasil.

\section{RESULTADOS}

A partir do exame de seleção, os médicos aprovados iniciam o treinamento básico, em serviço, durante um a dois anos, em Pediatria, com tempo variável para complementação em Neurologia. No terceiro e quarto anos, desenvolvem a programação voltada à Neurologia Infantil.

$\mathrm{Na}$ Tabela, apresentamos as 17 Instituições que mantêm ensino e treinamento em serviço de Neurologia Infantil, com informações sobre o número de vagas, duração dos programas e número de horas de treinamento por ano, representando parte dos dados contidos no protocolo.

Dessa forma, em dezembro de 1999, com revisão em abril do ano 2000, constatamos 6 (seis) Instituições com Residência Médica em Neurologia Infantil, credenciadas pela CNRM, que titulam anualmente 13 novos profissionais na especialidade. Por outro lado, verificamos 10 serviços com conteúdo programático semelhante ao exigido pelo CNRM, porém não oficialmente credenciados, mas que anualmente fornecem certificados a 12 novos profissionais. Um serviço se encontra em diligência (Hospital do Servidor Público do Estado de São Paulo). Dessa forma, no Brasil, são colocados, anualmente, no mercado de trabalho, 26 médicos especialistas em Neurologia Infantil.

\section{DISCUSSÃO}

Merece reflexão, o fato de haver 6 serviços credenciados, 10 não credenciados e, um em diligência; semelhante situação está sendo vivenciada em outras especialidades. Esta constatação merece, urgentemente, ser revisada, integrando democraticamente o pensamento das Sociedades Médicas e da CNRM visando a obtenção a médio prazo, do padrão de excelência na formação em Residência Médica em Neurologia Infantil. A duração do programa nas 17 diferentes Instituições, incluindo o pré-requisito em Pediatria ou Neurologia, variou de 3 a 4 anos. O número de horas de 
Tabela. Instituições com Residência em Neurologia Infantil.

\begin{tabular}{cccccc}
\hline $\mathrm{N}^{\mathbf{0}}$ & Instituição & Credenciada* & Vagas & Duração** & Hora/ano \\
\hline 01 & USP, São Paulo, SP & Sim & 03 & 04 & 2500 \\
02 & USP, Ribeirão Preto, SP & Sim & 02 & 04 & 2800 \\
03 & UNIFESP, São Paulo, SP & Sim & 02 & 03 & 2400 \\
04 & UNESP, Botucatú, SP & Sim & 02 & 04 & 2880 \\
05 & UNICAMP, Campinas, SP & Sim & 02 & 04 & 2880 \\
06 & UFRS, Porto Alegre, RS & Sim & 02 & 04 & 2850 \\
07 & HSPE, São Paulo, SP & Diligência & 01 & 03 & 1920 \\
08 & FCM, Santa Casa, SP & Não & 02 & 04 & 1920 \\
09 & UFRJ, Rio de Janeiro, RJ & Não & 02 & 03 & 2800 \\
10 & FIOCRUZ, Rio Janeiro, RJ & Não & 01 & 03 & 2880 \\
11 & HB,Distrito Federal, DF & Não & 01 & 03 & 2880 \\
12 & FHMG, Belo Horizonte, MG & Não & 01 & 04 & 2762 \\
13 & UF Paraná, Curitiba, PR & Não & 01 & 04 & 1900 \\
14 & PUC Paraná, Curitiba, PR & Não & 01 & 03 & 2000 \\
15 & PUCRS, Porto Alegra, RS & Não & 01 & 03 & 2880 \\
16 & FFFCM, Porto Alegre, RS & Não & 01 & 04 & 2500 \\
17 & IMIP, Pernambuco, PE & Não & 01 & 04 & 2880 \\
\hline
\end{tabular}

*Credenciada pela CNRM

**Em anos incluindo o pré-requisito

USP- Universidade de São Paulo-São Paulo, SP

USP- Faculdade de Medicina de Ribeirão Preto, SP

UNIFESP- Universidade Federal de São Paulo(Escola Paulista de Medicina),São Paulo, SP

UNESP- Universidade Estadual de São Paulo, Botucatu, SP

UNICAMP- Universidade Estadual de Campinas, Campinas, SP

UFRS-Universidade Federal do Rio Grande do Sul, Porto Alegre, RS

HSPE- Hospital do Servidor Público do Estado de São Paulo-São Paulo, SP

FCM-Faculdade de Ciências Médicas da Santa Casa de São Paulo, São Paulo, SP

UFRJ- Universidade Federal do Rio de Janeiro, Rio de Janeiro, RJ

FIOCRUZ-Fundação Oswaldo Cruz, Rio de Janeiro, RJ

HB- Hospital de Base-Distrito Federal, DF

FH-Fundação Hospitalar Belo Horizonte, Belo Horizonte, MG

UFPR-Universidade Federal do Paraná, Curitiba, PR

PUC- Pontifícia Universidade Católica, Curitiba, PR

PUCRS-Pontifícia Universidade Católica de Porto Alegre, RS

FFFCM- Fundação Faculdade Federal Ciências Médicas de Porto Alegre, RS

IMIP- Instituto Materno Infantil-Recife, PE

treinamento por ano variou de 1900 a 2880 horas, distribuídas nas várias atividades em enfermarias, unidades de terapia intensiva, pronto socorro, berçários, laboratórios para estudo de líquidos orgânicos e líquido céfalorraquano, eletrofisiologia clínica, imagem, neuropatologia, entre outros. Os médicos residentes desenvolvem, também, estudos teórico-práticos em neuroanatomia, neurofisiologia, conhecimentos atualizados em temas de Neurologia Pediátrica, seminários, reuniões bibliográficas, clínico-patológicas e, conjuntas, com equipes de profissionais de outras especialidaes. 
Em relação à distribuição das instituições credenciadas pela CNRM pode ser reconhecido que na região Sudeste, com população de 68065975 habitantes, se localizam 10 centros de treinamento (5 serviços credenciados, 4 não credenciados e um em diligência); na região Sul, com 23862664 habitantes, 5 serviços, sendo, apenas, um credenciado; no Nordeste com população de 45334385 habitantes e, Centro Oeste, com população de 10769249 habitantes, apenas um centro de formação, em cada região, porém não credenciados. Atualmente,estão sendo submetidos a avaliação, 4 centros (PUC-Hospital Pequeno Principe em Curitiba, no Paraná; FioCruz; Rio de Janeiro; UFRJ-Hospital Martagão Gesteira no Rio de Janeiro e PUC-Porto Alegre, Rio Grande do Sul).

Um procedimento desejável seria observar a adequação do número de vagas ao mercado de trabalho e, também, a monitorização dos titulados para os locais de origem, procurando oferecer, por exemplo, dados relacionados ao número de especialistas por um milhão de habitantes. Foi possível constatar que 12 programas são desenvolvidos e mantidos em escolas médicas públicas, federais e estaduais, que, também, implantaram e desenvolveram Cursos de Pós-Graduação, sensoestrito, (Mestrado e Doutorado) $)^{1,2}$ obedecendo metas que visam promoção e diferenciação em conhecimentos e pesquisas.

As referidas escolas médicas, por sua vez, se localizam mais especificamente nos estados de São Paulo, Rio de Janeiro, Minas Gerais e Rio Grande do Sul, levando a uma aglutinação de profissionais, equipamentos sociais, estrutura e infra-estrutura nesses referidos estados da União. Por sua vez,o desenvolvimento econômico cultural em ciências e tecnologia, também, está polarizado particularmente no Centro-Oeste e Sul, nas especialidades médicas e em áreas de saúde, porém, não médicas $^{2,4}$. É inegavel e básico que a educação médica, envolvendo a Residência, a diferenciação nos conhecimentos e, as pesquisas, constituem investimento essencial que reverte diretamente em benefício da população como um todo.

Os dados aquí apresentados demonstram parte de um estudo mais amplo, que está em andamento, visando o melhor conhecimento sobre a Residência Médica em Neurologia Infantil em nosso país, procurando identificar as dificuldades no transcorrer da formação e agilisar as necessárias providências corretivas.

Assim foi possivel identificar os centros credenciados, ou não, pela CNRM, verificar o número de profissionais anualmente titulados, bem como a conjunção da distribuição dos serviços de Residência em Neurologia Infantil com a aglutinação de profissionais da área da saúde, nos estados do Sudeste e Sul.

\section{REFERÊNCIAS}

1. Moura-Ribeiro MVL, Funayama CAR. Requisitos mínimos de um Programa de Residência Médica: competências em Neurologia Infantil. FUNDAP, Documento de Trabalho. São Paulo, 1991.

2. Moura-Ribeiro MVL. A formação do Neurologista Infantil: passado, presente e futuro. Neuro-Press (São Paulo): 1998:2(2).

4. Conselho Federal de Medicina, Federação Nacional dos Médicos, Associação Médica Brasileira, Fundação Oswaldo Cruz. Perfil dos médicos do Brasil, vol. 5, São Paulo, 1995.

5. Lott IT, Ouvrier R. Training and research issues in child neurology: an international perspective. International Child Neurology Association Symposium: San Francisco, October 5, 1994.

3. Souza SEM. Residência em Neurologia no Brasil: levantamento em 1992. Relatório apresentado no XV Congresso Brasileiro de Neurologia da Academia Brasileira de Neurologia,

6. Lana-Peixoto MA. Residência médica e o título de especialista em neurologia. Arq Neuropsiquiatr 1989;47:503-505.

7. Lana-Peixoto MA. O papel da Academia Brasileira de Neurologia: uma visão pessoal Arq Neuropsiquiatr 1991;49:475-479. 